\title{
Formation and structure of ionomer complexes from grafted polyelectrolytes
}

\author{
Agata M. Brzozowska • Karel J. Keesman • \\ Arie de Keizer • Frans A. M. Leermakers
}

Received: 7 November 2010 /Revised: 19 December 2010 /Accepted: 23 December 2010 /Published online: 16 March 2011

(C) The Author(s) 2011. This article is published with open access at Springerlink.com

\begin{abstract}
We discuss the structure and formation of Ionomer Complexes formed upon mixing a grafted block copolymer (poly(acrylic acid)- $b$-poly(acrylate methoxy poly(ethylene oxide)), $\mathrm{PAA}_{21}-b-\mathrm{PAPEO}_{14}$ ) with a linear polyelectrolyte (poly(N-methyl 2-vinyl pyridinium iodide), P2MVPI), called grafted block ionomer complexes (GBICs), and a chemically identical grafted copolymer (poly(acrylic acid)-co-poly(acrylate methoxy poly(ethylene oxide)), $\mathrm{PAA}_{28}$-co-PAPEO ${ }_{22}$ ) with a linear polyelectrolyte, called grafted ionomer complexes (GICs). Light scattering measurements show that GBICs are much bigger $(\sim 70$ $100 \mathrm{~nm}$ ) and GICs are much smaller or comparable in size $(6-22 \mathrm{~nm})$ to regular complex coacervate core micelles (C3Ms). The mechanism of GICs formation is different from the formation of regular C3Ms and GBICs, and their size depends on the length of the homopolyelectrolyte. The sizes of GBICs and GICs slightly decrease with temperature increasing from 20 to $65^{\circ} \mathrm{C}$. This effect is stronger for GBICs than for GICs, is reversible for GICs and GBIC$\mathrm{PAPEO}_{14} / \mathrm{P}_{2} \mathrm{MVPI}_{228}$, and shows some hysteresis for GBIC-PAPEO $_{14} / \mathrm{P}_{2} \mathrm{MVPI}_{43}$. Self-consistent field (SCF)
\end{abstract}

A. M. Brzozowska • A. de Keizer • F. A. M. Leermakers Laboratory of Physical Chemistry and Colloid Science, Wageningen University,

Dreijenplein 6,

6703 Wageningen, the Netherlands

\author{
A. M. Brzozowska $(\bowtie)$ \\ Wetsus, Centre of Excellence for Sustainable Water Technology, \\ Agora 1, P.O. Box 1113, 8900 Leeuwarden, the Netherlands \\ e-mail: agata.brzozowska@wur.nl
}

\section{K. J. Keesman}

Systems and Control Group, Wageningen University,

Bornse Weilanden 9,

6708 Wageningen, the Netherlands calculations for assembly of a grafted block copolymer (having clearly separated charged and grafted blocks) with an oppositely charged linear polyelectrolyte of length comparable to the charged copolymer block predict formation of relatively small spherical micelles $(\sim 6 \mathrm{~nm})$, with a composition close to complete charge neutralization. The formation of micellar assemblies is suppressed if charged and grafted monomers are evenly distributed along the backbone, i.e., in case of a grafted copolymer. The very large difference between the sizes found experimentally for GBICs and the sizes predicted from SCF calculations supports the view that there is some secondary association mechanism. A possible mechanism is discussed.

Keywords Ionomer complexes · Grafted block ionomer complexes · Grafted ionomer complexes · Light scattering

\section{Introduction}

Regular complex coacervate core micelles (C3Ms), also known as "block ionomer complexes" [1] or "polyion complex micelles" [2], are formed upon mixing linear, oppositely charged polyelectrolytes of which at least one contains a neutral block. In solution, the assembly is driven by electrostatic attraction between the charged blocks and the entropy gain upon releasing the counterions. Charged blocks form a complex coacervate core which is stabilized by the neutral blocks, forming the corona. Upon addition of the homopolymer to the oppositely charged diblock copolymer, charged, loose structures with low aggregation numbers, so-called soluble complex particles (SCPs) are initially formed. At a critical composition neutral C3Ms are formed, and the number of SCPs decreases to zero at the preferred micellar composition (PMC). For regular C3Ms, 
the PMC corresponds to the point of complete charge neutralization [3]. A detailed description of the formation of C3Ms may be found elsewhere [4]. The stability of C3Ms in solution depends on the relative lengths of the charged and neutral blocks $[4,5]$, the salt concentration, and the $\mathrm{pH}$ $[4,6]$. The stability and structure of the C3Ms in the bulk, in turn, determine stability and structure of the adsorbed $\mathrm{C} 3 \mathrm{M}$ layer, as well as its functionality with respect to suppressing protein adsorption [6]. The assembly of linear and that of non-linear oppositely charged polyelectrolytes (i.e., grafted block or grafted copolymers) is rather similar. However, due to the presence of the short side chains, which make the copolymer less flexible, the resulting aggregates may not have the distinct core-corona structure, characteristic for regular C3Ms. Therefore, to avoid any misunderstanding, the term "grafted block ionomer complexes" (GBICs) is adopted for particles formed with a grafted block copolymer (Fig. 1a) and the term "grafted ionomer complexes" (GICs) is used for particles formed with a grafted copolymer (Fig. 1b).

Additional interactions may contribute to the aggregation process if, for instance, hydrophobic end-groups of sufficient length are present in the polyelectrolyte chains forming the micelles. Such polyelectrolytes may exhibit surfactant-like properties. In general, surfactants and polymers may form mixed aggregates. In solution, at low surfactant concentration, these coexist as separate species, but at the critical aggregation concentration (CAC) the surfactants start to aggregate with the polymer, which then acts as a nucleation core, until the saturation concentration is reached. As a result the surfactants will form multiple spherical micelles, located at fixed distances along the polymer chain [7]. If the polymer carries a charge opposite to that of a surfactant-like polyelectrolyte, aggregation is driven by both electrostatic attraction and hydrophobic interactions.

The objective of this study is to characterize the structure of (1) aggregates formed upon mixing a grafted block

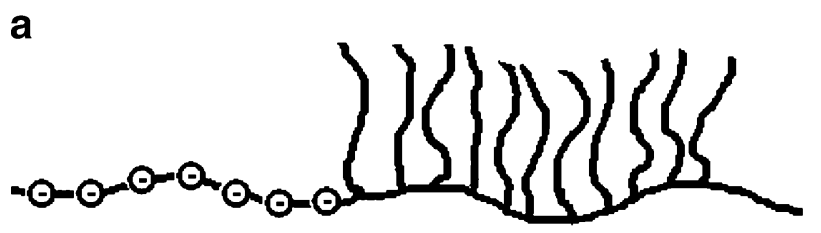

b

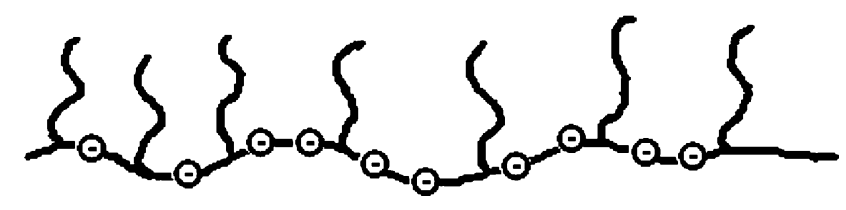

Fig. 1 A schematic representation of a grafted block copolymer (a) and a grafted copolymer (b) copolymer, $\mathrm{PAA}_{21}-b-\mathrm{PAPEO}_{14}$ and an oppositely charged polyelectrolyte, P2MVPI (GBICs), and (2) aggregates formed upon mixing a grafted copolymer, $\mathrm{PAA}_{28}-\mathrm{CO}$ $\mathrm{PAPEO}_{22}$ and oppositely charged polyelectrolytes, P2MVPI and $\mathrm{PAH} \cdot \mathrm{HCl}$ (GICs). Upon adsorption on solid-liquid interfaces GBICs and GICs suppress adsorption of proteins, provided that appropriate lengths of the charged blocks were chosen $[8,9]$. Adsorption of GBICs consisting of oppositely charged blocks of comparable lengths (22 and 43 monomers) resulted in full suppression of protein adsorption, whereas adsorption of GBICs consisting of charged blocks of significantly different lengths (22 and 228 monomers) resulted in enhanced protein adsorption.

\section{Experimental characterization of the aggregates}

In solution polyelectrolyte aggregates may be characterized by light scattering techniques. Dynamic light scattering (DLS) measurements allow determination of the decay constant $\Gamma_{\mathrm{DLS}}$. The results are analyzed using the method of cumulants. Measurements of $\Gamma_{\mathrm{DLS}}$ allow determination of the diffusion coefficient $D$ :

$\Gamma_{\mathrm{DLS}}=D q^{2}$

The scattering vector $q[1 / \mathrm{cm}]$ is given by the angle of detection $\theta$, the solvent refractive index $n$, and the wavelength $\lambda_{0}[\mathrm{~cm}]$ as:

$q=\frac{4 \pi n}{\lambda_{0}} \sin \left(\frac{\theta}{2}\right)$

The hydrodynamic radius $\left(R_{\mathrm{h}}\right)$ of the particles can be calculated using the Stokes-Einstein equation:

$D=\frac{k T}{6 \pi \eta_{\mathrm{s}} R_{\mathrm{h}}}$

Here $k\left[\mathrm{~m}^{2} \mathrm{~kg} /\left(\mathrm{s}^{2} K\right)\right]$ is the Boltzmann constant, $T[K]$ the temperature, $\eta_{\mathrm{s}}\left[\mathrm{m} \mathrm{kg} / \mathrm{s}^{2}\right]$ the solvent viscosity, and $R_{\mathrm{h}}[\mathrm{m}]$ the hydrodynamic radius of the particle.

Static light scattering (SLS) measurements provide information about the apparent molar mass of the particles $(M)$, the apparent micellar aggregation number $\left(N_{\text {agg }}\right)$, radius of gyration $\left(R_{\mathrm{g}}\right)$, the second virial coefficient $B$, and, for sufficiently large objects, the shape of the particles. The Rayleigh ratio $R\left(\theta, C_{\text {particle }}\right)$ which is a measure of the scattering power of a dispersion related to the concentration of scattering objects, is defined as:

$R\left(\theta, C_{\text {particle }}\right)=\frac{I_{\text {ex }}}{I_{\text {toluene }}} R(\theta)_{\text {toluene }}$

where $C_{\text {particle }}\left[\mathrm{g} / \mathrm{cm}^{3}\right]$ is the concentration of the scatterers (aggregates), $I_{\mathrm{ex}}=I_{\text {sample }}-I_{\text {solvent }}$ is the excess scattered intensity, $I_{\text {toluene }}$ is the scattering intensity of the reference 
solution (toluene), and $R(\theta)_{\text {toluene }}$ is the Rayleigh ratio of the reference solution. $R(\theta)_{\text {toluene }}$ is equal to $3.2 \times 10^{-5}[1 /$ $\mathrm{cm}]$ at room temperature.

The Rayleigh ratio allows for molecular characterization of the scattering particles according to:

$\frac{K C_{\text {particle }}}{R(\theta)}=\frac{1}{M} \frac{1}{P(q)} \frac{1}{S(q)}=\left(\frac{1}{M}+2 B C_{\text {particle }}\right) \frac{1}{P(q)}$

where $P(q)$ is the form factor, $S(\mathrm{q})$ is the structure factor, $M[\mathrm{~g} / \mathrm{mol}]$ is the molecular mass of the scatterers, $B$ $\left[\mathrm{cm}^{3} \mathrm{~mol} / \mathrm{g}^{2}\right]$ is the second virial coefficient, and $K$ is an optical constant. $S(\mathrm{q})$ is equal to unity at sufficiently low particles concentrations where interactions between the scattering particles may be neglected. $K\left[\mathrm{~cm}^{2} \mathrm{~mol} / \mathrm{g}^{2}\right]$ is defined as follows:

$K=\frac{4 \pi^{2} n^{2}}{N_{\mathrm{AV}} \lambda_{0}^{4}}\left(\frac{\mathrm{d} n}{\mathrm{~d} c}\right)^{2}$

where $n$ is refractive index of the solvent, $N_{\mathrm{AV}}[1 / \mathrm{mol}]$ is Avogadro's number, $\lambda_{0}[\mathrm{~cm}]$ is the wavelength of the incoming beam, and $\mathrm{d} n / \mathrm{d} c\left[\mathrm{~cm}^{3} / \mathrm{g}\right]$ is the refractive index increment of the solute.

The form factor, $P(\mathrm{q})$, expresses the effect of particle shape and size on the scattering power. For scatterers with dimensions (in terms of the radius of gyration $R_{\mathrm{g}}$ ) much smaller than the wave length of the incident light $\left(q R_{\mathrm{g}} \rightarrow 0\right)$, the form factor $P(\mathrm{q})$ is independent of the shape of the particles, and may be approximated by:

$P(q)=1-\frac{q^{2}}{3} R_{g}^{2}+\ldots$

$\frac{K C_{\text {particle }}}{R(\theta)}=\left(\frac{1}{M}+2 B C_{\text {particle }}\right)\left(1+\frac{q^{2}}{3} R_{\mathrm{g}}^{2}\right)$

Eq. 8 is the basic equation for the Zimm plot in which $M$ is determined by extrapolation of $K C_{\text {particle }} / R$ to $q=0$ and $C_{\text {particle }}=0 . R_{\mathrm{g}}$ and $B$ follow from the slope of $K C_{\text {particle }} / R$

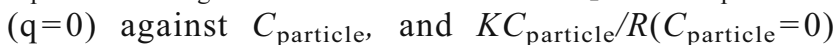
against $q^{2}$. The measured scattering intensity of sufficiently small particles and low particle concentrations may also be analyzed in terms of the Guinier approximation (Eq. 9):

$\frac{K C_{\text {particle }}}{R\left(\theta, C_{\text {particle }}\right)} \approx \frac{1}{M} \exp \left(\frac{1}{3} q^{2} R_{\mathrm{g}}^{2}\right)$

If the size of the scattering particles becomes comparable to the length of the incident light $\left(1 \leq q R_{\mathrm{g}} \leq 10\right)$ higher terms in the power series expansion of the form factor (Eq. 7) become important and the full equation has to be applied. For these objects the form factor allows distinguishing between different particle shapes. For a homo- geneous hard sphere with radius $R$, the form factor $P(\mathrm{q})$ is given in Ref. [10]:

$P(q, R)=F(q, R)^{2}=\left(\frac{3[\sin (q R)-q R \cos (q R)]}{(q R)^{3}}\right)^{2}$

where $F(q)$ is an amplitude of the form factor. The form factor for an ellipsoid of revolution was determined by Guinier [11], and expressed for three semi-axes $R, R, \varepsilon R$, and angle $\alpha$ :

$P(q, R, \varepsilon)=\int_{0}^{\frac{\pi}{2}} F[q, r(R, \varepsilon, \alpha)]^{2} \sin (\alpha) \mathrm{d} \alpha$

where

$r(R, \varepsilon, \alpha)=R\left(\sin ^{2}(\alpha)+\varepsilon^{2} \cos ^{2}(\alpha)\right)^{\frac{1}{2}}$

Thus, for low concentrations, the combined Eqs. 5 and 10 describe light scattering for an object with a shape approximated by a homogeneous hard sphere, and Eq. 5 and Eqs. 11 and 12 describe light scattering for an object approximated by an ellipsoid of revolution.

For known molecular weight of the building block [4], the aggregation number of the Ionomer Complexes can be estimated by:

$N_{\text {agg }}=\frac{M}{M_{\text {building_block }}}$

The molecular weight of the building block is defined as the sum of the molecular weight of the block copolymer and the molecular weight of the stoichiometric amount of oppositely charged polyelectrolyte minus the molecular weight of the corresponding number of counterions of both polymers [4]. Calculated values of $M_{\text {building_block }}$ are summarized in Table 1.

\section{Self-consistent field calculations}

Self-consistent field (SCF) modeling allows for a detailed molecular description of the polymeric micelles. Existing models examine classical micellar systems formed by copolymers in selective solvents [12-15]. In these models, the formation of micelles is described by a driving force,

Table 1 Calculated values of molecular weights of building blocks

\begin{tabular}{lc}
\hline System & $M_{\text {building_block }}(\mathrm{g} / \mathrm{mol})$ \\
\hline GBIC-PAPEO $_{14} / \mathrm{P}_{2} \mathrm{MVPI}_{43}$ & $1.18 \times 10^{4}$ \\
GBIC-PAPEO $_{14} / \mathrm{P}_{2} \mathrm{MVPI}_{228}$ & $1.19 \times 10^{4}$ \\
GIC-PAPEO $_{22} / \mathrm{P}_{2} \mathrm{MVPI}_{43}$ & $1.68 \times 10^{4}$ \\
GIC-PAPEO $_{22} / \mathrm{PAH}_{160}$ & $1.47 \times 10^{4}$ \\
\hline
\end{tabular}


typically the immiscibility of one of the blocks with the solvent resulting in formation of a core, and a stopping mechanism, commonly the build-up of the pressure between the solvated corona chains. The assembly of oppositely charged polyelectrolyte (PE) chains, leading to the formation of complex coacervate core micelles (C3Ms), was also recently studied $[16,17]$. It was argued that some hydrophobic attraction in the PE blocks may also contribute to the inter-polyelectrolyte complexation. In these early studies the electrostatic interactions were mimicked by nearest-neighbor attractions, implemented by a negative Flory-Huggins interaction parameter, $\chi$.

Realistic information about the thermodynamic stability and the structure of the aggregates is available through SCF modeling provided that the set of parameters accurately describes the system, as there is a strong relation between molecular architecture, strength of driving and stopping forces, and the structure of the most-likely micelles. Typical predictions are in a good agreement with experimentally determined concepts from surfactant science. For instance, spherical micelles were found to be stable, relative to worm micelles when the size of the core is smaller than that of the corona.

SCF modeling starts with a mean-field free energy in a preset coordinate system. Here, we focus on a spherical coordinate system, because the molecular structure points to the stability of spherical micelles. The equations are implemented using lattice approximations. The lattice sizes are of length $b=0.3 \mathrm{~nm}$. For each molecular species, the free energy includes a single-chain partition function. This quantity is usually evaluated using the freely jointed chain approximation. The electrostatic interactions are accounted for on the Poisson-Boltzmann level. The non-electrostatic interactions are evaluated with the Bragg-Williams approximation and described by the well-known Flory-Huggins parameter. Typically, it is assumed that the system is incompressible. The SCF solution, which appears as a saddle point of the free energy, is found up to high precision by an appropriate numerical (iterative) scheme [18-20].

\section{Materials and methods}

Poly(2-vinyl pyridine) $\left(\mathrm{P}_{2} \mathrm{VP}_{43} ; M_{\mathrm{n}}=4.15 \mathrm{~kg} / \mathrm{mol}, \mathrm{PDI}=\right.$ 1.09) was purchased from Polymer Standard Service (Mainz, Germany). Poly(N-methyl 2-vinyl pyridinium iodide) $\left(\mathrm{P}_{2} \mathrm{MVPI}_{228} ; M_{\mathrm{w}}=56 \mathrm{~kg} / \mathrm{mol}, \mathrm{PDI}=1.09\right)$ was purchased from Polymer Source Inc, Canada. Poly(acrylic acid)-b-poly (acrylate methoxy poly(ethylene oxide)) ( $\mathrm{PAA}_{21}-b$ $\mathrm{PAPEO}_{14} ; M_{\mathrm{n}}=8.86 \mathrm{~kg} / \mathrm{mol}$, PDI $=1.4$, the molecular mass of graft is $M_{\text {graft }}=0.450 \mathrm{~kg} / \mathrm{mol}$ corresponding to a length of 8-9 monomers per grafted PEO chain), and poly(acrylic acid)-co-poly(acrylate methoxy poly(ethylene oxide)) $\left(\mathrm{PAA}_{28}-\mathrm{co}-\mathrm{PAPEO}_{22} ; M_{\mathrm{n}}=8.86 \mathrm{~kg} / \mathrm{mol}, P D I=1.4, M_{\text {graft }}=\right.$ $0.450 \mathrm{~kg} / \mathrm{mol}$, also corresponding to $8-9$ monomers per grafted PEO chain) were a kind gift from Christophe Detrembleur, Université de Liège. The synthesis and characterization of these polymers are described in detail elsewhere [21]. Schematic representations of the polymeric molecules used in this study are shown in Figs. 2, 3, and 4. Poly(allylamine hydrochloride) ( $\mathrm{PAH} \cdot \mathrm{HCl}_{160} ; M_{\mathrm{n}}=15 \mathrm{~kg} /$ mol, $\geq 95 \%$ ), sodium chloride $(\mathrm{NaCl})$, sodium hydroxide $(\mathrm{NaOH}, 1 \mathrm{M})$, hydrochloric acid $(\mathrm{HCl}, 1 \mathrm{M})$, and toluene of analytical grade were purchased from Sigma. All chemicals were used as received.

\section{Quaternization of P2VP}

$\mathrm{P}_{2} \mathrm{VP}_{43}$ was quaternized and subsequently characterized according to the procedure described in detail elsewhere $[8,9]$.

\section{Characterization of GBICs and GICs in solution}

Stock solutions of $\mathrm{PAA}_{21}-b-\mathrm{PAPEO}_{14}, \mathrm{PAA}_{28}-c o-\mathrm{PAPEO}_{22}$, P2MVPI $228, \mathrm{P}_{2} \mathrm{MVPI}_{43}$, and PAA $\cdot \mathrm{HCl}_{160}$ in MilliQ water were prepared. Prior to the measurements, solutions of the required polymer and salt concentrations were prepared from the stock solutions. The $\mathrm{pH}$ was adjusted to $7 \pm 0.1$
Fig. 2 A schematic representation of $\mathrm{PAA}_{21}-b$-PAPEO ${ }_{14}$, including a hydrophobic end-group $\mathrm{C}_{12} \mathrm{H}_{25}$ being a residue of the primer used during the synthesis of this grafted block copolymer

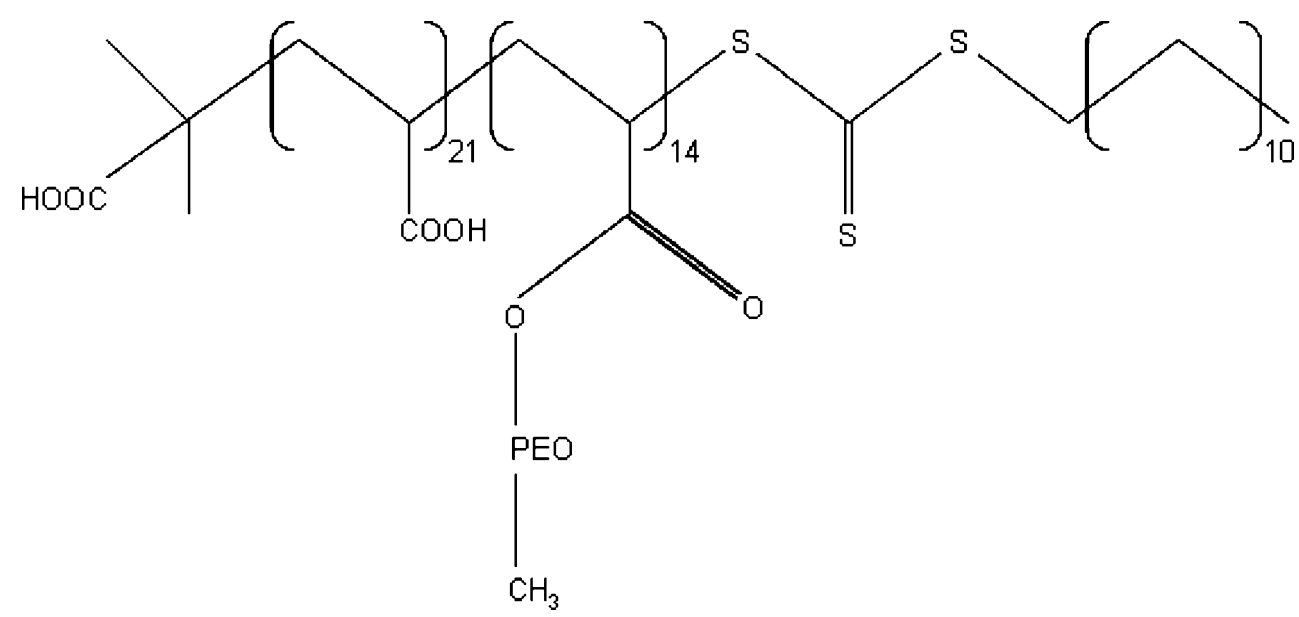


Fig. 3 A schematic representation of $\mathrm{PAA}_{28}-\mathrm{co}-\mathrm{PAPEO}_{22}$ molecule. Subscripts denote numbers of repeating blocks: acrylic acid: $x \times m=28$ and acrylate methoxy poly(ethylene oxide): $x \times n=22$. The hydrophobic end-group, $\mathrm{C}_{12} \mathrm{H}_{25}$, is a residue of the primer used during the synthesis of this grafted copolymer

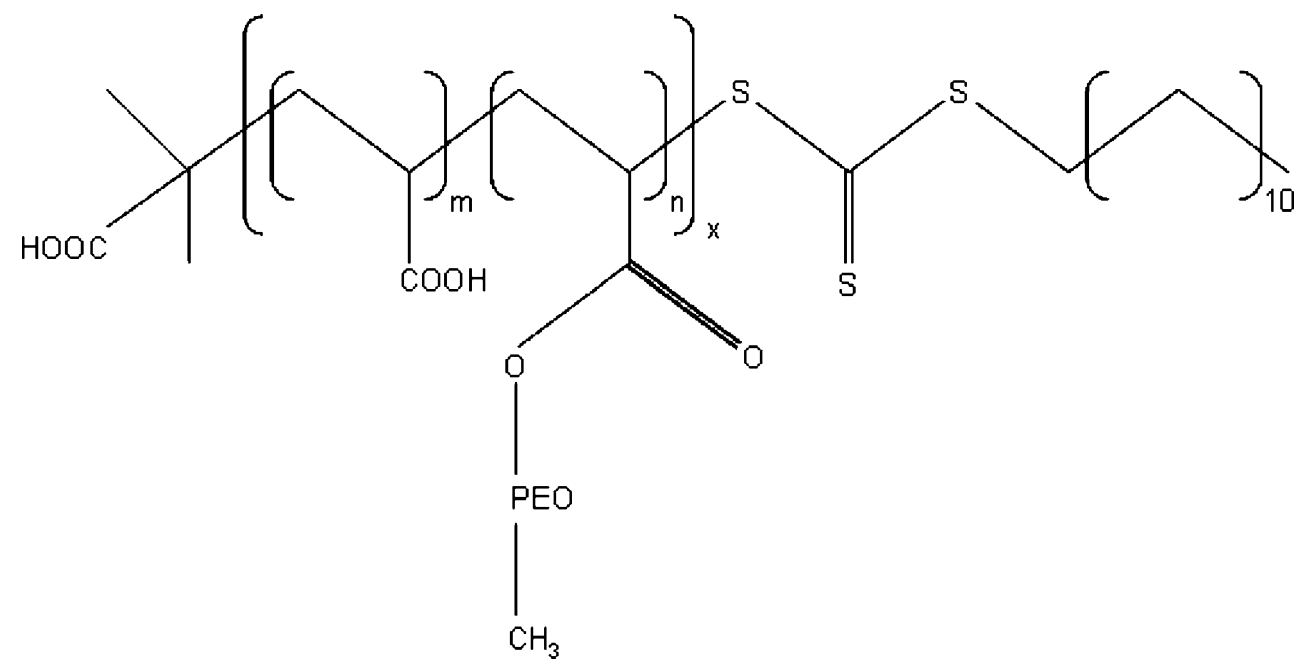

with 1 or $0.1 \mathrm{M} \mathrm{NaOH}$ and $\mathrm{HCl}$, when necessary. GBICs solutions were prepared at the PMC as determined with light scattering titrations [9], and GICs solutions were prepared at the point of zero charge determined with zeta potential measurements[8]. All solutions were prepared in $10 \mathrm{mM} \mathrm{NaCl}, \mathrm{pH}$ 7. The concentrations of GBICPAPEO $_{14} / \mathrm{P}_{2} \mathrm{MVPI}_{43}$ and GBIC-PAPEO ${ }_{14} / \mathrm{P}_{2} \mathrm{MVPI}_{228}$ were $0.1 \mathrm{~g} / \mathrm{l}$. The concentrations of GIC-PAPEO $22 /$ $\mathrm{P}_{2} \mathrm{MVPI}_{43}$ and GIC-PAPEO $22 / \mathrm{PAH}_{160}$ vary from 2 to $0.25 \mathrm{~g} / \mathrm{l}$. GICs solutions were filtered with $0.45 \mu \mathrm{m}$ syringe filter prior to the measurements. Light scattering measurements were performed using an ALV light scattering instrument equipped with an ALV_500 digital correlator and a $300 \mathrm{~mW}$ argon ion laser $(532 \mathrm{~nm})$, at $25 \pm 0.5{ }^{\circ} \mathrm{C}$. Decalin was used as a refractive index matching medium. Experiments were performed approximately $12 \mathrm{~h}$ after mixing the polyelectrolytes at the PMC in order to ensure equilibrium. For each sample the scattering intensity was measured 10 times per angle $\theta=30^{\circ}$ to $120^{\circ}$, every $1^{\circ}$ (GBICs) and $\theta=30^{\circ}$ to $130^{\circ}$, every $5^{\circ}$ (GICs), in order to determine the Rayleigh ratio $R\left(\theta, C_{\text {particle }}\right)$.

\section{Results and discussion}

Grafted molecules discussed in this study (Figs. 2 and 3) are composed of the same number of chemically identical groups. The difference between the two is the distribution of charged and grafted monomers along the backbone of the molecule. In the grafted copolymer, PEO grafts are randomly distributed along the charged chain. In the grafted block copolymer the charged units and the neutral grafted units are organized in separate blocks. This difference has a dramatic impact on the structure of Ionomer Complexes. It is expected that upon mixing with oppositely charged polyelectrolytes the copolymers aggregate due to electro- static attraction between the oppositely charged chains. As, usually, charge compensation is obtained at the PMC (i.e., the maximum in the scattering intensity). The assembly may be further promoted by hydrophobic interactions between the hydrophobic end-groups attached to both copolymers (Figs. 2 and 3). However, this process depends on the accessibility of the hydrophobic chains, and thus on the structure of the aggregate. We expect that if the aggregates are formed with GBICs a core-shell structure is formed similar to regular $\mathrm{C} 3 \mathrm{Ms}$, but the hydrophobic end-groups are not (fully) hidden in this core-shell structure and extend beyond the corona-solvent interface. As a consequence, secondary assembly may take place in three dimensions. However, aggregates formed with GICs are rather stiff and small, terminated by a hydrophobic endgroup. As a result we expect that the secondary aggregation process due to the hydrophobic stickers is limited and will occur mainly in one dimension. To further validate these expectations, we investigate the stability of these aggregates as function of the total polymer concentration and the temperature.

The $R_{\mathrm{h}}$ of GBIC-PAPEO ${ }_{14} / \mathrm{P}_{2} \mathrm{MVPI}_{43}$ and GBIC$\mathrm{PAPEO}_{14} / \mathrm{P}_{2} \mathrm{MVPI}_{228}$ have been determined with DLS titrations, at $90^{\circ}$ detection angle, at the PMC and as a function of the micellar concentration after an equilibration time of about $12 \mathrm{~h}$. As shown in Fig. 5 (left) the observed radii of GBICs are much larger than values reported for

Fig. 4 A schematic representation of P2MVPI subscript $y$ corresponds to the number of monomers in the polymer chains used in the experimental part (43 and 228)

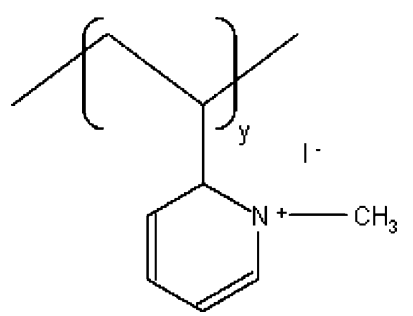


Fig. 5 Effect of the total polymer concentration on the hydrodynamic radii $\left(R_{\mathrm{h}}\right)$ of GBIC-PAPEO ${ }_{14} / \mathrm{P}_{2} \mathrm{MVPI}_{43}$ and (filled circles) GBIC-PAPEO ${ }_{22} /$ $\mathrm{P}_{2} \mathrm{MVPI}_{228}$ (left) and GIC$\mathrm{PAPEO}_{22} / \mathrm{P}^{2} \mathrm{MVPI}_{43}$ (filled circles) GIC-PAPEO $22 / \mathrm{PAH}_{160}$ (empty circles; right).

Experiments were carried out in $10 \mathrm{mM} \mathrm{NaCl}, \mathrm{pH} \mathrm{7}$, at room temperature, $\theta=90^{\circ}$. Samples were equilibrated for $12 \mathrm{~h}$ prior measurements

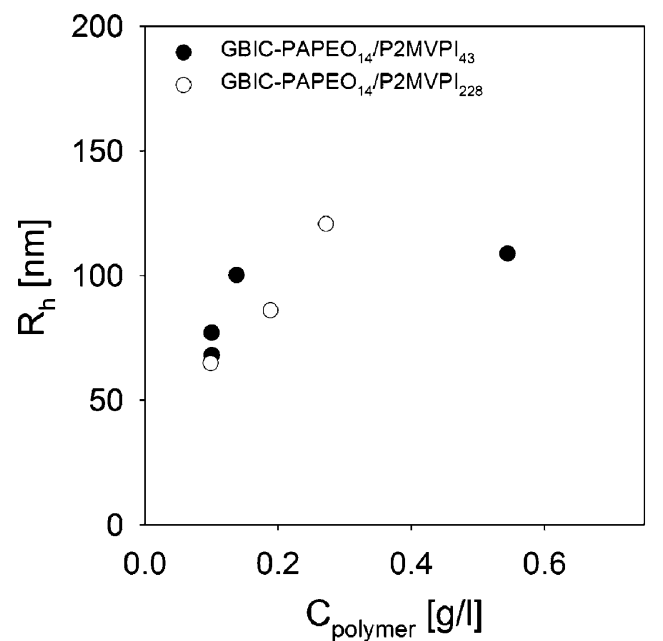

regular $\mathrm{C} 3 \mathrm{M}$ systems $[3,22]$. At low concentrations (c $0.1 \mathrm{~g} / \mathrm{l})$, values of $90-110 \mathrm{~nm}$ are obtained as compared with 20-30 for regular C3Ms [3, 22]. Such values are comparable to those reported in a previous paper [9] for concentrations between 0.1 and $0.4 \mathrm{~g} / \mathrm{l}$. In the low concentration range, the effect of concentration is relatively weak. However, at concentrations at 5.5 and $6 \mathrm{~g} / \mathrm{l}$ for GBIC-PAPEO ${ }_{14} / \mathrm{P}_{2} \mathrm{MVPI}_{43}$ and GBIC-PAPEO $14 /$ $\mathrm{P} 2 \mathrm{MVPI}_{228}$, respectively, we have observed radii of about 400 and $150 \mathrm{~nm}$, respectively (Fig. 6). We anticipate that the larger size of the micelles and the size increase at higher concentrations is a consequence of a secondary aggregation mechanism due to the hydrophobic sticker. However, the stopping mechanism maybe due to hiding of the stickers inside the aggregate upon further growth.

The $R_{\mathrm{h}}$ of GIC-PAPEO $22 / \mathrm{P}_{2} \mathrm{MVPI}_{43}$ and GIC-PAPEO $22 /$ $\mathrm{PAH}_{160}$ determined at the PMC during DLS titrations, at $90^{\circ}$, were much smaller, and were approximately 8 and $22 \mathrm{~nm}$, respectively [8]. The radius of GIC-PAPEO 22 $\mathrm{P}_{2} \mathrm{MVPI}_{43}$ is very small. We attribute this observation to
Fig. 6 Change of the hydrodynamic radius $\left(R_{\mathrm{h}}\right)$ and of scattering intensity divided by the total polymer concentration $(I / c)$ of GBIC-PAPEO $14 / \mathrm{P}_{14} \mathrm{MVPI}_{43}$ and GBIC-PAPEO 14

$\mathrm{P}_{2} \mathrm{MVPI}_{228}$ with temperature, at $\theta=90^{\circ}$. Experiments were carried out in $10 \mathrm{mM} \mathrm{NaCl}, \mathrm{pH} 7$. The total polymer concentrations were 5.45 and $6.01 \mathrm{~g} / 1$ for GBIC-PAPEO $_{14} / \mathrm{P}_{2} \mathrm{MVPI}_{43}$ and GBIC-PAPEO $14 / \mathrm{P}_{2} \mathrm{MVPI}_{228}$, respectively. Temperature change of $5{ }^{\circ} \mathrm{C}$ was obtained within $5 \mathrm{~min}$. Once the desired temperature was reached it was kept constant for the duration of the LS measurements (ten runs, $60 \mathrm{~s}$ each)

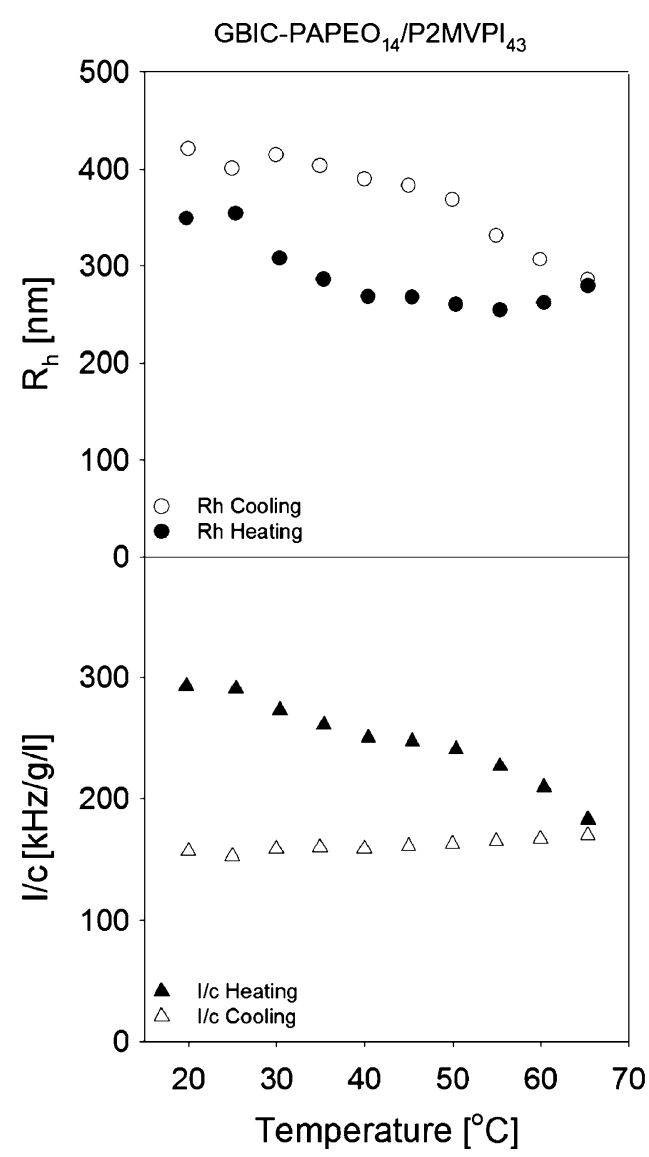




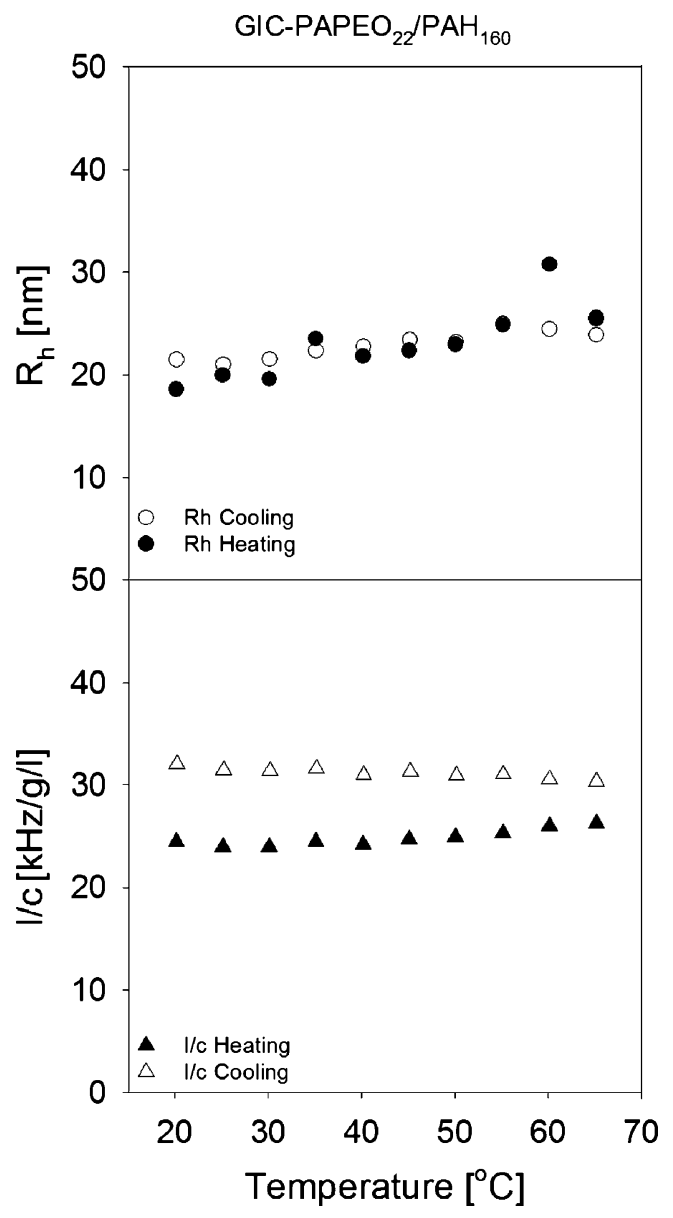

Fig. 7 Change of the hydrodynamic radius $\left(R_{\mathrm{h}}\right)$ and scattering intensity divided by the total polymer concentration $(I / c)$ of GIC$\mathrm{PAPEO}_{22} / \mathrm{PAH}_{160}$ with temperature, at $\theta=90^{\circ}$. Experiments were carried out in $10 \mathrm{mM} \mathrm{NaCl}, \mathrm{pH}$ 7. The total polymer concentration amounted to $2.05 \mathrm{~g} / \mathrm{l}$. Temperature change of $5{ }^{\circ} \mathrm{C}$ was obtained within $5 \mathrm{~min}$. Once the desired temperature was reached it was kept constant for the duration of the LS measurements (ten runs, $60 \mathrm{~s}$ each) the low aggregation number which is a consequence of the stiffness of the $\mathrm{PAA}_{28}$-co-PAPEO${ }_{22}$ molecule and relatively small lengths of the oppositely charged $\mathrm{P}_{2} \mathrm{MVPI}_{43}$ chain [8]. It was concluded that the size of a GICs is mainly determined by the length of the homopolymer, and not so much by the chemical properties of the homopolymers. For the GICs, the hydrodynamic radius is independent of concentration up to a concentration of $2 \mathrm{~g} / \mathrm{l}$. (Fig. 5, right). From these results, it follows that the effect of the hydrophobic terminating end-groups of the grafted copolymers does not lead to a significant three-dimensional growth.

In order to further investigate the contribution of the hydrophobic $\mathrm{C}_{12} \mathrm{H}_{25}$ groups to the aggregate formation, we have also studied the effect of temperature on (hydrodynamic) size. Results for GBICs are presented in Fig. 6. Here, we focus on the big aggregates at the highest concentration, in order to emphasize the effect of the (small) additional interactions due to the hydrophobic end-groups.

The hydrodynamic radii of the GBICs decrease as the temperature increases from 20 to $65{ }^{\circ} \mathrm{C}$. For GBIC$\mathrm{PAPEO}_{14} / \mathrm{P}_{2} \mathrm{MVPI}_{228}$, this apparent change in particle size and in the corresponding scattering intensity are entirely reversible. Changes of $R_{\mathrm{h}}$ for GBIC-PAPEO ${ }_{14} / \mathrm{P}_{2} \mathrm{MVPI}_{43}$ show some hysteresis, and the corresponding changes in scattering intensity are not fully reversible. From the results, we conclude that the high temperature partly disrupts the big aggregates at this high concentration, but the aggregates do not fall apart to the size at low GBICs concentrations (around $100 \mathrm{~nm}$ ), and certainly not to the primary micelles (which are expected to be as small as $\sim 10 \mathrm{~nm}$, see also the SCF modeling below). We speculate that the temperature effect is related to the nature of the aggregates at high concentration. At low concentrations we expect the effect will be absent or at least much smaller. However, we have not studied the temperature effect at low GBICs concentration separately.
Fig. 8 DLS results: $\Gamma_{D L S}$ and $R_{\mathrm{h}}$ as functions of $\mathrm{q}^{2}$ measured for solutions of GBIC$\mathrm{PAPEO}_{14} / \mathrm{P}_{2} \mathrm{MVPI}_{43} 12$ and $36 \mathrm{~h}$ after mixing two micellar components at the $\mathrm{PMC}$ in $10 \mathrm{mM} \mathrm{NaCl}, \mathrm{pH}$ 7. The total polymer concentration is $0.1 \mathrm{~g} / \mathrm{l}$. Solid lines in the graph on the left hand side correspond to linear fits of the experimental data
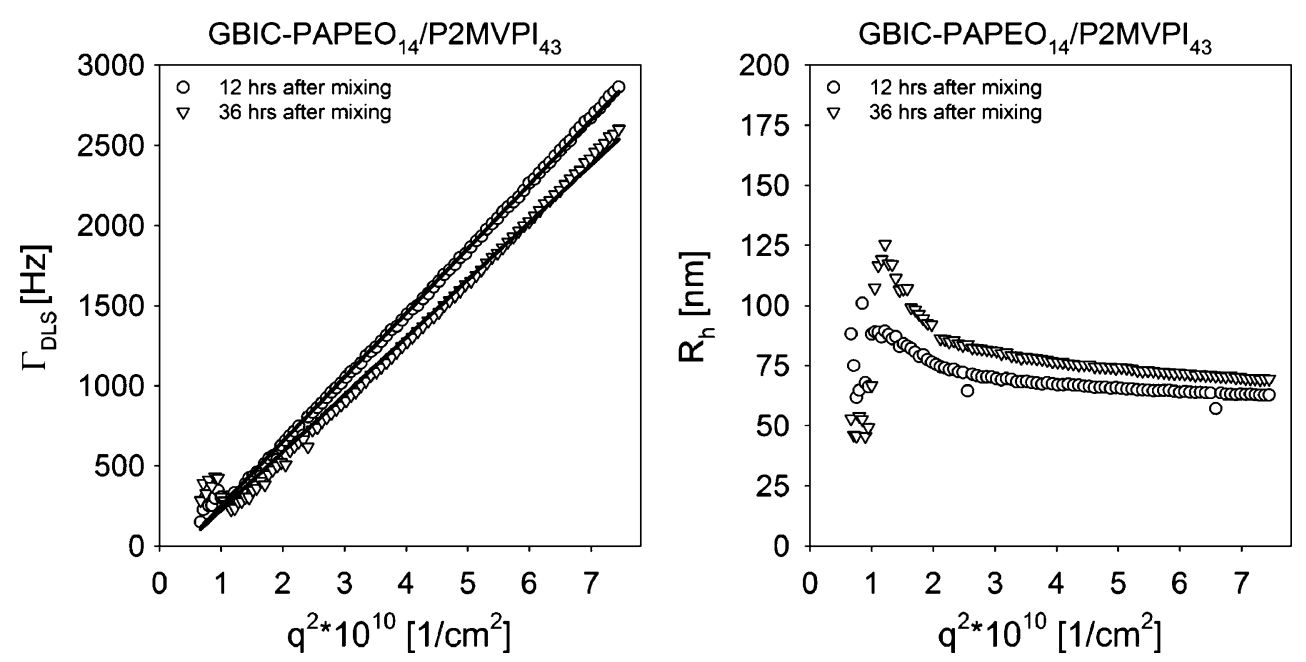
Fig. 9 DLS results: $\Gamma_{D L S}$ and $R_{\mathrm{h}}$ as functions of $q^{2}$ measured for solutions of GBIC-

$\mathrm{PAPEO}_{14} / \mathrm{P}_{2 \mathrm{MVPI}_{43}} 12$ and $36 \mathrm{~h}$ after mixing two micellar components at PMC in $10 \mathrm{mM}$ $\mathrm{NaCl}, \mathrm{pH}$ 7. Total polymer concentration, $0.1 \mathrm{~g} / 1$
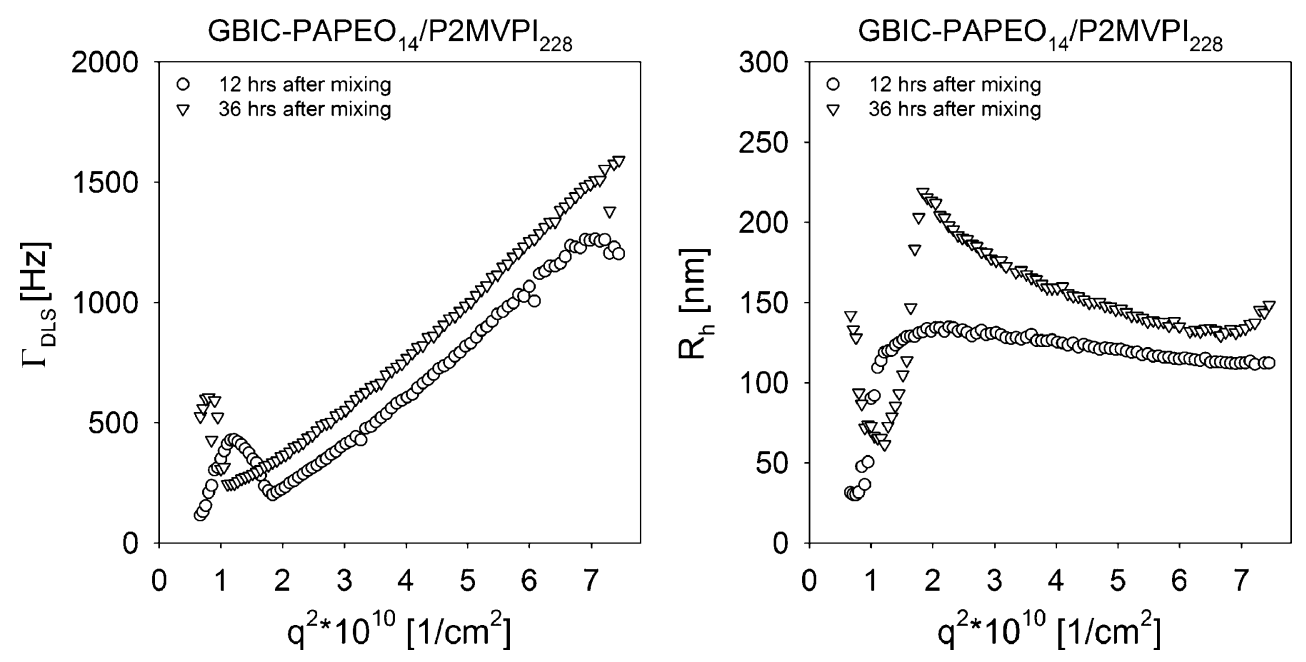

The temperature dependence for GICs is shown in Fig. 7. The size and the scattering intensity of GICs are not significantly influenced by the temperature. We observe only a small increase in size as the temperature increases. We relate this observation to the fact that electrostatic interactions between the charged blocks are independent of the temperature.

To obtain some information about the shape and the apparent molecular masses of GBICs and GICs we carried out additional light scattering measurements. Results presented in Figs. 8 and 9 show changes in the decay coefficient $\Gamma$ with $q^{2}$ and corresponding changes in $R_{\mathrm{h}}$. At low $q^{2}$ changes of $\Gamma$ with $q^{2}$ strongly deviate from linear, indicating polydispersity of the scattering objects or their non-spherical shape. The deviation is less pronounced for GBIC-PAPEO $_{14} /$ P2MVPI $_{43}$ than for GBIC-PAPEO $14 /$ $\mathrm{P} 2 \mathrm{MVPI}_{228}$.

For both systems we observe slow changes of the particle size and structure in time, in particular for GBIC$\mathrm{PAPEO}_{14} / \mathrm{P}_{2} \mathrm{MVPI}_{228}$. This is a clear indication that the system is not in the equilibrium.
Multi-angle light scattering measurements for GICPAPEO $_{22} / \mathrm{P}_{2} \mathrm{MVPI}_{43}$ and GIC-PAPEO $14 / \mathrm{PAH}_{160}$ show that for both systems the decay coefficient $\Gamma$ changes linearly with $q^{2}$ (Fig. 10). Consequently, the corresponding hydrodynamic radius depends only slightly on the angle of detection, indicating that GICs are most likely spherical structures with a narrow size distribution.

Information about the apparent molar masses $(M)$ and radii $(R)$ of GBIC-PAPEO ${ }_{14} / \mathrm{P}_{2} \mathrm{MVPI}_{43}$ and GBIC$\mathrm{PAPEO}_{14} / \mathrm{P}_{2} \mathrm{MVPI}_{228}$ were obtained by fitting Eq.5 to the experimental results. The dimensions of GBICs are comparable to the wavelength of the incident light $\left(R_{\mathrm{g}}>\lambda / 20=\right.$ $27 \mathrm{~nm}$ ), i.e., beyond Rayleigh and Guinier scattering regimes [23]. Therefore we applied the full equations for the form factor $P(\mathrm{q})$ for different shapes. To describe GBICs, we have chosen models for the form factor with the lowest number of adjustable parameters: a homogeneous and monodisperse hard sphere (Eqs. 5 and 10), and an ellipsoid of revolution (Eqs.5, 11, and 12). Model fitting was done by least squares minimization, starting from the average value of $R_{\mathrm{h}}$ (Matlab software). Results are shown in
Fig. 10 DLS results: $\Gamma_{D L S}($ left $)$ and $R_{\mathrm{h}}(r i g h t)$ as functions of $q^{2}$ measured for solutions of GIC$\mathrm{PAPEO}_{22} / \mathrm{P}_{2} \mathrm{MVPI}_{43}$ (filled circles) and GIC-PAPEO 22 / $\mathrm{PAH}_{160}$ (empty circles) in $1 \mathrm{mM}$ $\mathrm{NaCl}, \mathrm{pH}$ 7. Total polymer concentration, $2(\mathrm{~g} / \mathrm{l})$. A slight increase of $R_{\mathrm{h}}$ measured for GIC-PAPEO $22 / \mathrm{P}_{2} \mathrm{MVPI}_{43}$ is most probably a consequence of presence of larger aggregates in the solution. Solid lines in the left panel correspond to linear fits of the experimental data
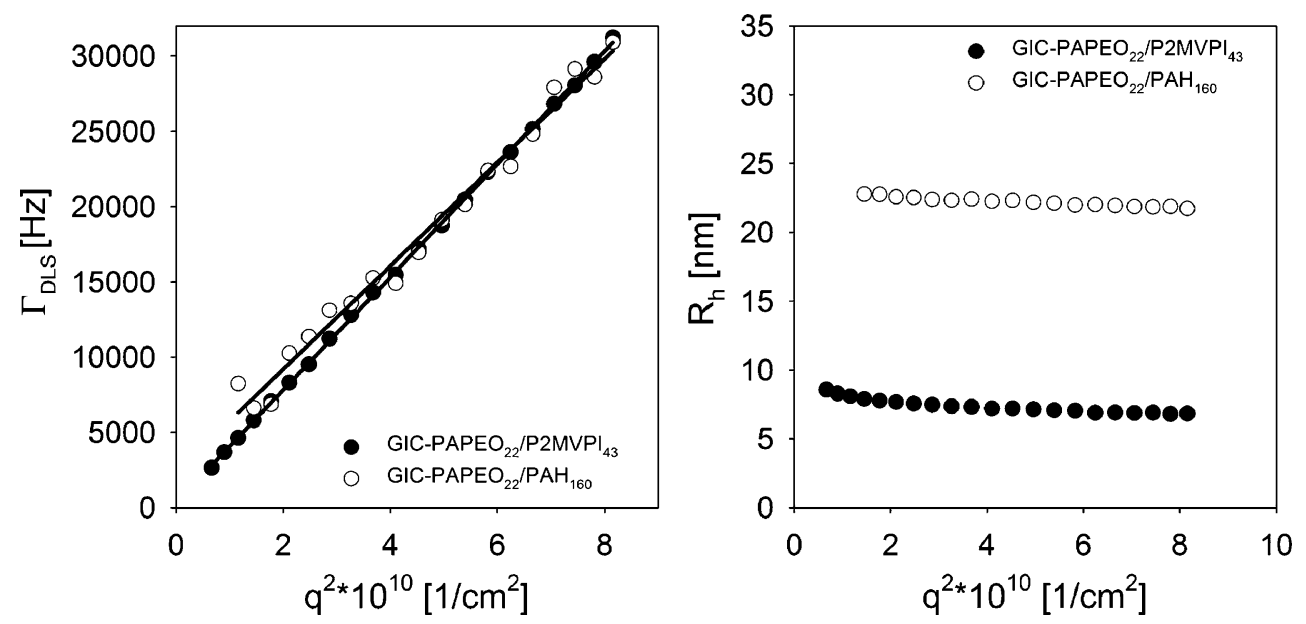
Fig. 11 Upper panels show results of fitting a model with the form factor for a homogeneous hard sphere (solid line) to the multi-angle light scattering data (empty circles) obtained for GBIC-PAPEO ${ }_{14} / \mathrm{P}_{2} \mathrm{MVPI}_{43} 12$ (left) and 36 (right) $\mathrm{h}$ after preparation of GBICs. The lower panels show the corresponding residues. Experiments were carried out at $10 \mathrm{mM} \mathrm{NaCl}, \mathrm{pH}$ 7. The most significant deviation between the model and the experimental data is observed at low scattering angles (low $q^{2}$ )

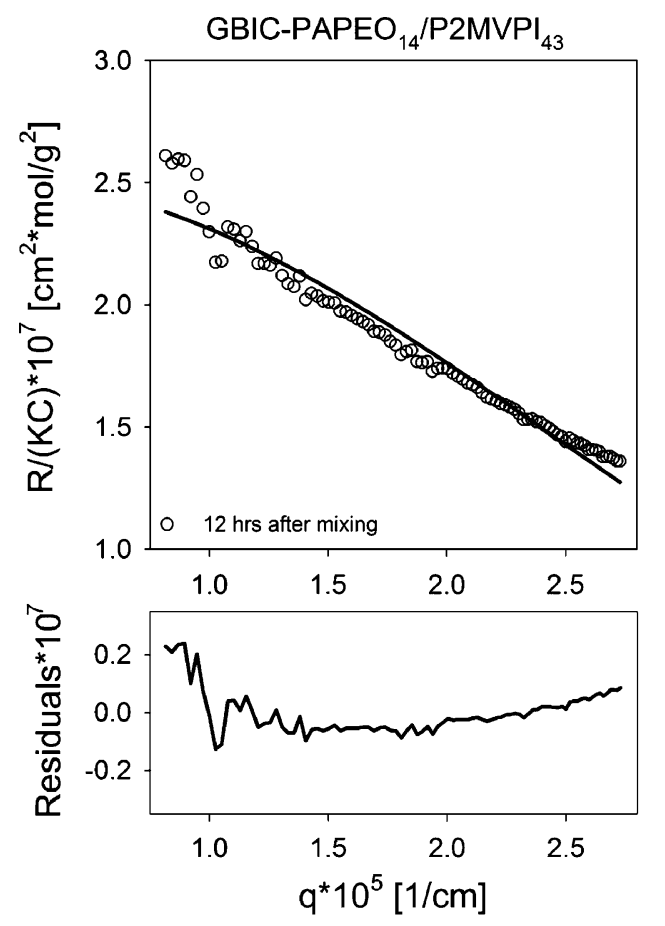

Figs. 11, 12, 13, and 14, and numerical results are summarized in Table 2.

The quality of the fit decreases at low scattering angles, where the data become less reliable. One may argue that the applied model does not describe the structures accurately. However, application of more complex models (i.e., ellipsoid of revolution, tri-axial ellipsoid, data not shown) and adding polydispersity does not improve the fit. Moreover, the models become insensitive to some of the parameters. In addition to the experimental errors the nonideal fit may also result from the fact that GBICs have not reached the state of the equilibrium yet, i.e., are changing in time. Figure 12 shows the sensitivity of the model for GBIC-PAPEO ${ }_{14} / \mathrm{P}_{2} \mathrm{MVPI}_{43}$ to changes in model parameters, namely molecular weight $(M)$ and radius $(R)$. Partial derivatives of the model equation with respect to each parameter are plotted against $q$. The larger the slope of the curve (the absolute value of the partial derivative) at a given point, the more sensitive the model is to small changes of the parameter at this point. In Fig. 12, values on the left and right ordinates result from the scaling applied during the model fitting procedure. The model describing GBICs (measured $36 \mathrm{~h}$ after mixing) shows a relatively high and constant sensitivity of $K C_{\text {particle }} / R(\theta)$ with respect to $R$ at high scattering angles $\left(q>2.510^{-5} 1 / \mathrm{cm}\right)$. In general, the sensitivities increase with increasing $q$ allowing a more accurate estimation of $M$ and $R$ at higher values of $q$.

Accurate fitting of GBIC-PAPEO $14 / \mathrm{P}_{2} \mathrm{MVPI}_{228}$ data (12 $\mathrm{h}$ after mixing) was found difficult and inaccurate at low scattering angles (low $q^{2}$, Fig. 13, left panel). A much
Fig. 12 Sensitivity of the fitted model with respect to both model parameters: molar mass of the scattering aggregate $(M)$ and radius of the aggregate $(R)$, obtained for GBIC-PAPEO $14 /$ $\mathrm{P}_{2} \mathrm{MVPI}_{43} 12$ (left) and 36 (right) $\mathrm{h}$ after preparation of GBICs
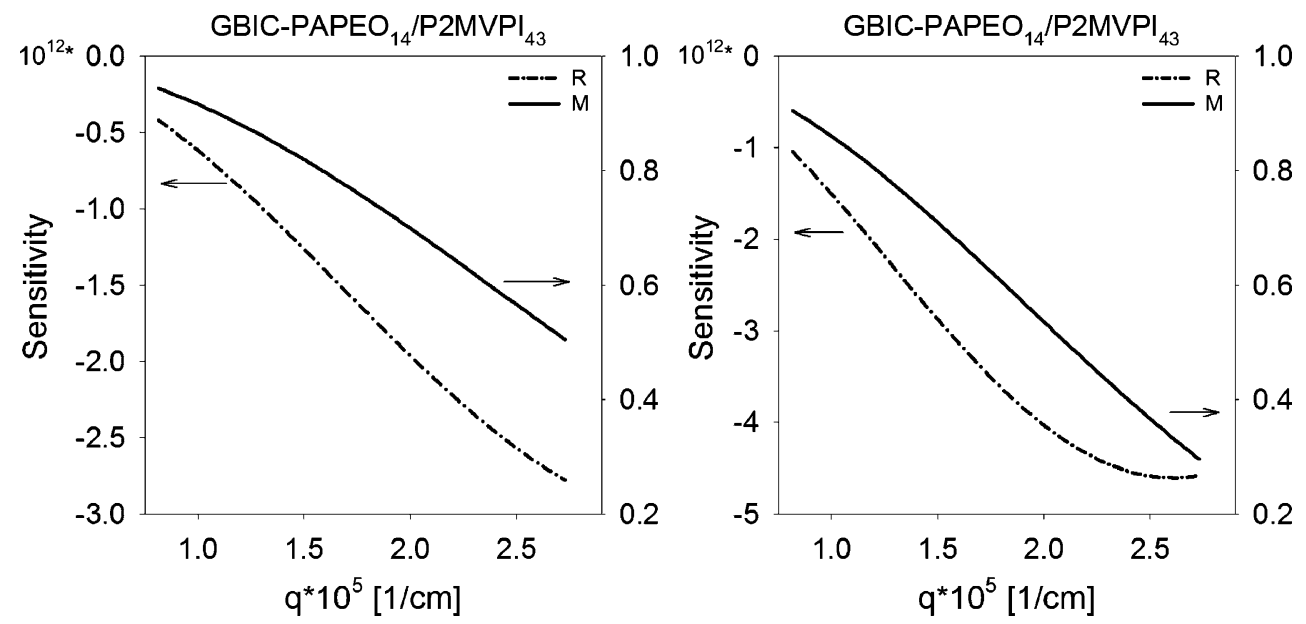
Fig. 13 The upper panels show results of fitting a model with the form factor for a homogeneous hard sphere (left) and an ellipsoid of revolution (right; solid line) to the multi-angle light scattering data (empty circles) obtained for GBIC$\mathrm{PAPEO}_{14} / \mathrm{P}^{2} \mathrm{MVPI}_{228} 12$ (left) and 36 (right) $\mathrm{h}$ after preparation of GBICs. The lower panels show the corresponding residues. Experiments were carried out at $10 \mathrm{mM} \mathrm{NaCl}$, $\mathrm{pH}$ 7. The most significant deviation between the model and the experimental data is observed at low scattering angles (low $q^{2}$ ) for GBICs $12 \mathrm{~h}$ after preparation

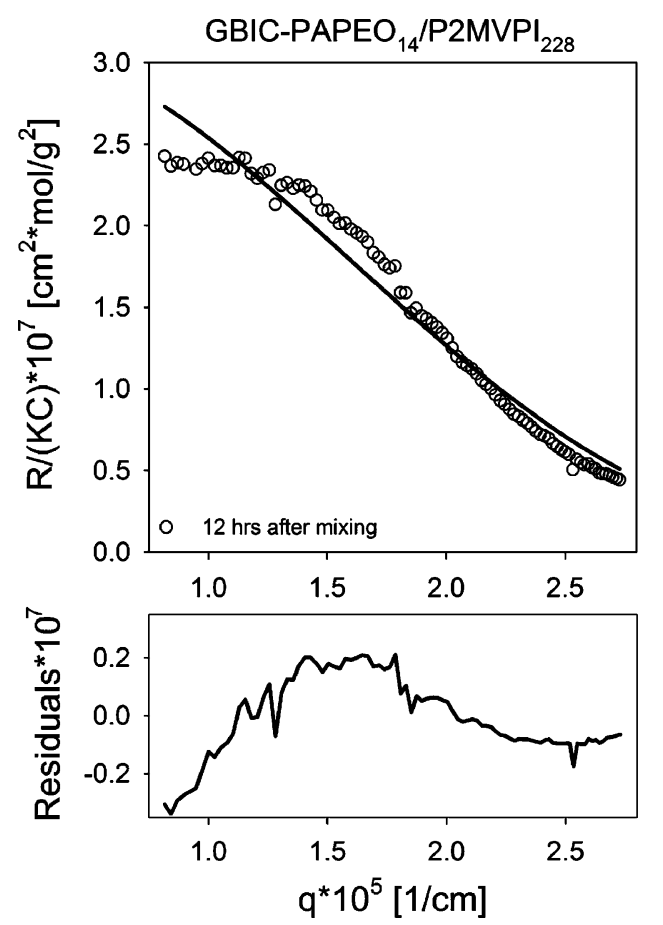

better model fit was obtained for these particles for data measured $36 \mathrm{~h}$ after preparation (Fig. 13, right panel). Herewith, we find a form factor for an ellipsoid of revolution. In Fig. 14, we summarize the sensitivity of the model for GBIC-PAPEO ${ }_{14} / \mathrm{P}_{2} \mathrm{MVPI}_{228}$ to changes in $M, R$, and the $\varepsilon$. Interestingly, for ellipsoid of revolution we find a model which sensitivity with respect to $R$ and $\varepsilon$ decrease with increasing $q$ (Fig. 14, left panel).

The results summarized in Table 2 indicate significant changes in sizes of the aggregates over time. The radii determined for GBIC-PAPEO $14 / \mathrm{P}^{2} \mathrm{MVPI}_{43}$ are in a good agreement with experimentally determined $R_{\mathrm{h}}$ (Fig. 8). The radius estimated for GBIC-PAPEO ${ }_{14} / \mathrm{P}_{2} \mathrm{MVPI}_{228} 36 \mathrm{~h}$ after mixing is significantly larger than the one estimated from DLS measurements at $\theta=90^{\circ}$ (Fig. 9). The molar masses of GBICs indicate very high aggregation numbers, exceeding the values expected for regular C3Ms. Both the dimensions and the aggregation numbers of GBICs indicate that they are composed of smaller sub-micelles assembled due to additional hydrophobic interactions between the hydrophobic end-groups of grafted block copolymers.

The scattering from small GIC-PAPEO $22 / \mathrm{P} 2 \mathrm{MVPI}_{43}$ and GIC-PAPEO $22 / \mathrm{PAH}_{160}$ particles, is within the Rayleigh and Guinier scattering regimes [23] so that the form factor $P(\mathrm{q})$ can be approximated with Eq. 7. The information about the molar mass and $R_{\mathrm{g}}$ of the particle can be obtained from experimental data applying Zimm and/or Guinier approximations, Eqs.11 and 12, respectively.

The $M, N_{\mathrm{agg}}$, and micellar hydrodynamic radii extrapolated to zero angle $\left(R_{\mathrm{h}}{ }^{0}\right)$ determined with DLS and SLS are summarized in Table 3. In Fig. 15, we plot the Guinier
Fig. 14 Sensitivities of the fitted models with respect to all model parameters: molar mass of the scattering aggregate $(M)$, the radius of the aggregate $(R)$, and the radius scaling factor $(\varepsilon)$ obtained for GBIC-PAPEO 14 P2MVPI $_{228} 12$ (left) and 36 (right) hours after preparation of GBICs
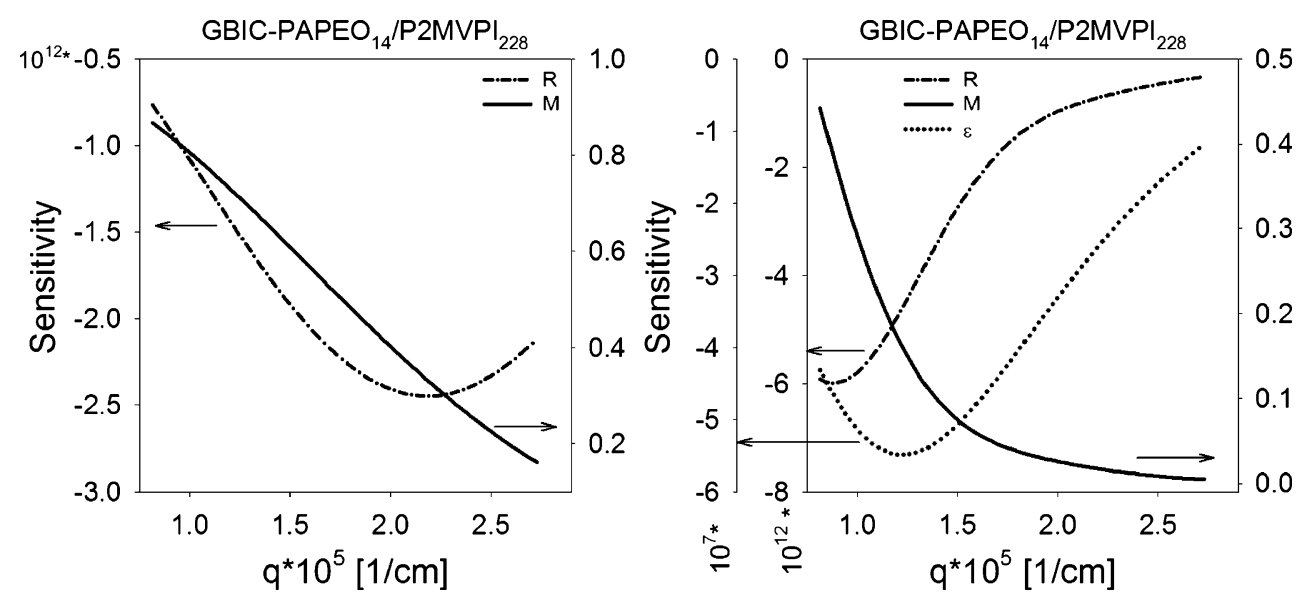
Table 2 Summary of the model fitting to the experimental SLS and DLS data

\begin{tabular}{lcccccc}
\hline System & Time $(\mathrm{h})$ & $R(\mathrm{~nm})$ & $R_{\mathrm{h}, 90^{\circ}}$ & $M \times 10^{5}(\mathrm{~g} / \mathrm{mol})$ & $N_{\mathrm{agg}}$ & $\varepsilon$ \\
\hline GBIC-PAPEO $_{14} / \mathrm{P}^{2} \mathrm{MVPI}_{43}$ & 12 & $66.1 \pm 0.7$ & 65.9 & $252 \pm 2$ & 2,145 \\
& 36 & $86.3 \pm 0.9$ & 73.8 & $496 \pm 6$ & 4,217 & - \\
GBIC-PAPEO $_{14} /$ P2MVPI $_{22}$ & 12 & $103 \pm 1$ & 120.7 & $315 \pm 5$ & 2,679 & - \\
& 36 & $294 \pm 2$ & 145.5 & $2,400 \pm 30$ & 20,202 \\
\hline
\end{tabular}

$R$ radius of the aggregate, $R_{h}$ hydrodynamic radius determined at $90^{\circ}$ from DLS measurements, $M$ molecular mass of the aggregate, $N_{\text {agg }}$ aggregation number calculated from Eq. 13, $\varepsilon$ radius scaling factor

approximation of the measured data. According to Eq. $9, R_{\mathrm{g}}$ can be determined from the slope of the Guinier plot. However, the change of $R\left(\theta, C_{\text {particle }}\right)$ as a function of angle of detection $(q)$ was only minor. For GIC-PAPEO $22 / \mathrm{PAH}_{160}$ the slope is approximately zero, and for GIC-PAPEO 22 $\mathrm{P}_{2} \mathrm{MVPI}_{43}$ it is slightly negative. This kind of behaviour is expected for particle sizes within the Rayleigh limit, i.e., particles much smaller than the wavelength of the incoming beam $\left(R_{\mathrm{h}}{ }^{0}<\lambda / 20=27 \mathrm{~nm}\right)$. In this regime, the particles may be considered as point scatterers and there is practically no information about the particle dimensions. Thus $R_{\mathrm{g}}$ values are not reported.

\section{Self-consistent field calculations}

The polymers forming GBICs are modeled taking into account their structural features on the monomer level. Electrostatic interactions are introduced by assigning charges to the polyelectrolyte blocks forming the micellar core. Other interactions are treated by means of FloryHuggins parameters. In Table 4, we present the relevant details. The partial positive charge "P" in the 2-vinyl pyridinium (2VP) monomer is distributed over the "ring" (mimicked by a double-branched side group); the degree of quaternization of P2MVPI (85\%) is also taken into account here. The negative charge of the acrylic acid monomer is located in the carboxyl group and we modeled this as $\mathrm{CN}_{2}$ where "N" represents a negative charge of -0.5 .

The calculations were performed in a semi-open ensemble. This means that the system was closed for the homopolyelectrolyte chain(s) and open for the solvent, the ions, and the oppositely charged copolymer with a neutral block. By

Table 3 Summary of the light scattering results

\begin{tabular}{lll}
\hline & GIC-PAPEO $_{22} / \mathrm{P}_{2} \mathrm{MVPI}_{43}$ & GIC-PAPEO $_{22} / \mathrm{PAH}_{160}$ \\
\hline$R_{\mathrm{h} 0}(\mathrm{~nm})$ & 8 & 22 \\
$M(\mathrm{~g} / \mathrm{mol})$ & $1.31 \times 10^{5}$ & $6.14 \times 10^{5}$ \\
$N_{\mathrm{agg}}$ & 8 & 42 \\
\hline
\end{tabular}

$R_{h}{ }^{0}$ hydrodynamic radius extrapolated to zero angle, $M$ molar mass of GICs estimated from Zimm approximation choosing a fixed number of homopolymers in the calculations, the aggregation number for a particular (sub)calculation is fixed. For each generated SCF solution, we first check the thermodynamic parameters. There exists only a small range of aggregation numbers for which the micelles pass all the thermodynamic stability tests [24-27] and only these results are further analyzed. Following the experimental systems, we focus on systems above the critical micellization concentration and for which the micellar concentration is dilute (no micelle-micelle interaction). We found thermodynamically stable micelles at low ionic strength. Their composition is very close to the point of zero charge, i.e., there are almost equal amounts of positive and negative charges in the micellar core.

Figure 16 shows the radial volume fraction profile for a micelle with grand potential (work of formation) $\Omega \sim 10 \mathrm{kT}$. This energy must be balanced back by the translation entropy of the micelle. This implies, in this case, a (dilute) micellar volume fraction of $\varphi_{\mathrm{m}} \sim 10^{-5}$. At this micellar concentration, the most-likely micelle carries seven cationic

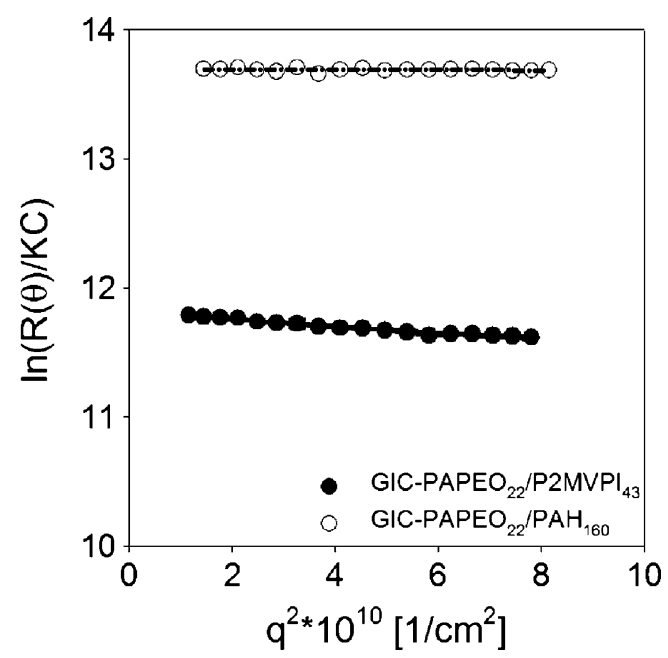

Fig. 15 The LS results: Guinier plot. Experiment was carried out in $1 \mathrm{mM} \mathrm{NaCl}, \mathrm{pH}$ 7. The total polymer concentration was $2 \mathrm{~g} / \mathrm{l}$. Measurements at lowest angles $\left(\theta=30^{\circ}\right.$ and $\left.35^{\circ}\right)$ have been excluded from analysis due to a very high measured values, probably due to the presence of larger aggregates in a solution. Lines represent the linear fits to the experimental data 
Table 4 Information about the molecular model used in the SCF calculations

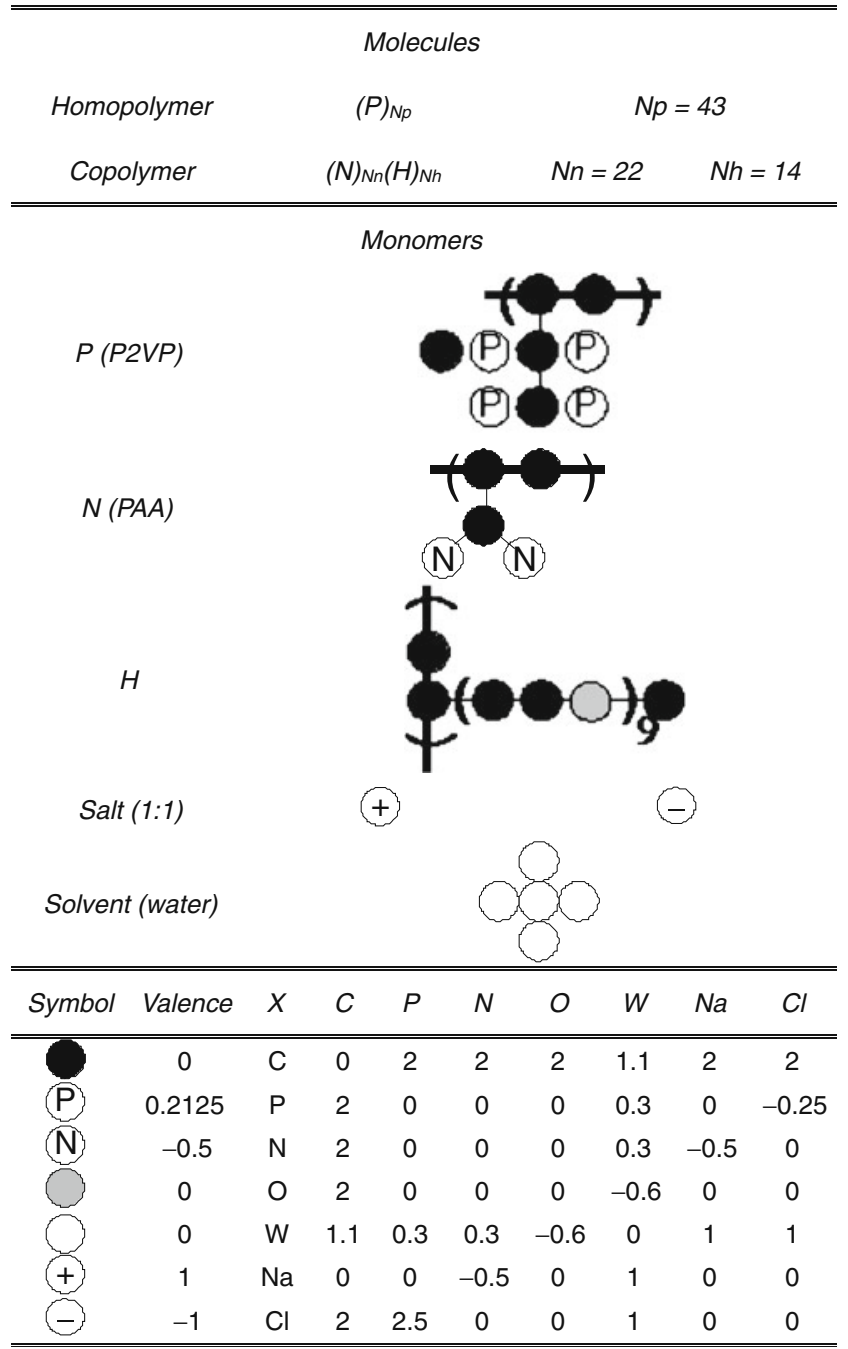

The top part contains an overview of the overall molecular structures of the (chain) molecules. In the second part, we show the chemical architectures of the monomers, water, and ions. In the schemes, the thick solid line represents the backbone. In the side chains, thin solid lines are used to indicate the bonds. The last part contains the names, valences, symbols of the monomers, and the Flory-Huggins interaction parameters. This parameter set is in part based on calculations of classical surfactant self-assembly [29] (these specify the important $\chi_{\mathrm{CW}}, \chi_{\mathrm{OW}}$ values). Furthermore, we implemented an effective repulsion between $\mathrm{C}$ and charged segments, and some non-ideal mixing of the salt ions with water (W). Finally, $\mathrm{Na}$ and $\mathrm{Cl}$ were given some additional attractive interactions to the oppositely charged segments of the polyelectrolytes in such a way that the partition coefficients are matched

homopolymers and on average just over 11 anionic copolymers to ensure the charge compensation. The micellar core resulting from the applied parameters is well hydrated. However, the experimental estimates for the water content of $\mathrm{C} 3 \mathrm{Ms}$ can be up to several tens of percent [28]. This relatively low water content must be attributed to the relatively large number of hydrophobic $\mathrm{C}$ segments in the blocks forming the core. The correlation attraction between positively and negatively charged core blocks is neglected in this Poisson-Boltzmann-like treatment of electrostatics. The radial volume fractions in Fig. 16 show that there is a large overlap between the core and the corona forming segments. This overlap is significantly larger than for classical surfactants. The low interfacial tension between core and solvent is due to the broad interface.

From Fig. 16 (left panel), the radius of the micelle can be estimated. Here, one should decide on some definition of how to compute the radius, but even inspection by eye already shows that a reasonable upper limit amounts to 20 lattice layers. This corresponds to a radius of approximately $6 \mathrm{~nm}$. This radius is much smaller than the one extracted from the light scattering measurements for this system. We will return to this issue in the discussion below. Figure 16 (right panel) shows the profile of the electrostatic potential in combination with the radial charge distribution across the micelle. For the discussed system both quantities are small. This is consistent with an approximately electrically neutral micellar core. Even though the charge distribution is non-trivial, the magnitude of the charge separation in the micelle is so small that further discussion of these distributions is not meaningful.

The radial profiles of the volume fraction of the small ions (Fig. 17; right ordinate) follow the Boltzmann weight, in which the electrostatic potential is an important contribution. The micelles contain a slightly higher amount of polycations than polyanions; the concentration of anions in the core is higher than of cations, and the potential tends to be positive in this case.

As indicated earlier, the micelle discussed in Figs. 16 and 17 is the most likely structure for a given micellar concentration. Other micellar structures that carry fewer or more polycations occur as fluctuations. The width of this distribution can be estimated from the corresponding grand potentials of such micelles. We do not show this analysis, but mention that the results indicate that fluctuations in aggregation numbers are limited to plus or minus two to three homopolymer chains. Hence, the micellar size distribution is relatively sharp for this system.

We performed a corresponding analysis, using the same parameter set, for the system with a five times longer polycation chain. In the SCF model the size of the micelle is controlled by crowding of the corona chains. Therefore, the formation of similar micelles with almost the same composition and a very similar amount of cationic segments (hence a five times decrease in number of chains) was predicted. In reality, the number of chains can only vary by integer values, and therefore we expect unusual fluctuations in the size of the micelle in this case.

The micellization process was dramatically inhibited when the PEO side chains were distributed homogeneously 
Fig. 16 Left, radial volume fraction $\varphi(r)$ profiles for water (thin dotted line), the cationic homopolymer (dashed line), and both blocks of the anionic copolymer (solid lines). Both the core and the corona regions are indicated. Right, the corresponding electrostatic potential profile $\psi[V]$ (left ordinate), and the dimensionless charge distribution $q / e$ (right ordinate)

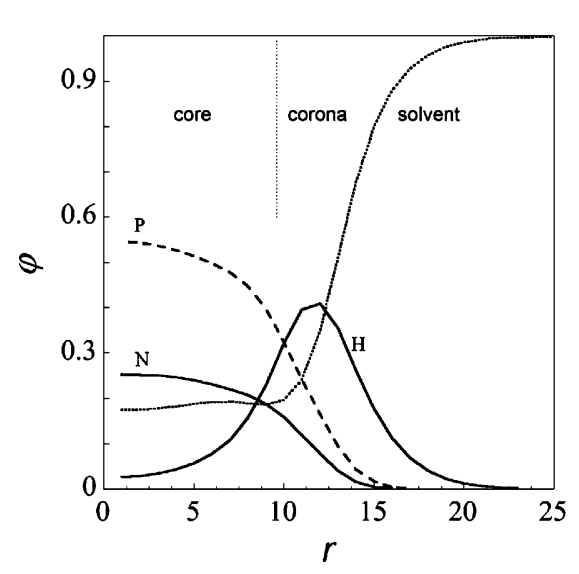

along the anionic polymer backbone. This finding is in good agreement with the results presented in Fig. 10 for GIC-PAPEO $22 / \mathrm{P}^{2} \mathrm{MVPI}_{43}\left(R_{\mathrm{h}} \sim 8 \mathrm{~nm}\right)$. In this particular case, the distribution of PEO probably also had a gradient. These results show that in order to form stable micelles it is important to separate the core forming block from the corona forming block in the molecular architecture.

One may argue that the small micellar sizes predicted by the SCF model are an artifact introduced by the values of parameters implemented to describe the system. A small change in the parameter value may have severe consequences for the micellar structure. For instance, modeling the ions as more hydrophilic may result in their complete exclusion from the micellar core, and as a result the stability of the micelles becomes insensitive to the ionic strength. The set of parameters applied to describe GBICs predicts disintegration of the aggregates as the salt concentration increases, which is in agreement with the experimental results [9]. In the discussed model, hydrophobic interactions are partly responsible for the stability of the micelles. Making the monomers more hydrophobic may result in exclusion of more solvent from the micellar core. One may argue that a more hydrated core will increase its size and possibly destabilize the spherical geometry. However, a reduction of the core density typically implies a lower strength of the driving force for micellization. A reduction of the driving force leads to a reduction of the aggregation numbers. The low aggregation numbers point again to the spherical micelle as the first choice of assembly.

The size of the micelles can be increased by reduction of the solvent quality for PEO. For nonionic surfactants, this behavior is well known. In our polymers, we have an equivalent of almost 130 EO segments and such a PEO chain can be easily dissolved in water. The segments of the non-polar backbone may change this picture and may give the anionic copolymer a relatively strong temperature dependent solubility characteristic. Another factor which may explain the strong difference between the sizes found by light scattering [9] and the SCF results discussed above is the presence of a hydrophobic moiety in the anionic copolymer chain. This group may trigger a secondary assembly of the micelles into super aggregates, as suggested by Fig. 13 where we observed a significant difference between the shapes after 12 and $36 \mathrm{~h}$. We cannot include this effect in our modeling, because hierarchical assemblies are out of scope of the current version of the SCF model.

\section{Conclusions}

We have shown that change in a distribution of charged and neutral groups along the backbone of the grafted copolymer PAA- $b(c o)-P A P E O$, containing a hydrophobic end-group, has a significant impact on the formation and the structure of the described ionomer complexes. Aggregates formed with a GBICs are much bigger than regular C3Ms $\left(R_{\mathrm{h}} \sim 70-100 \mathrm{~nm}\right)$, whereas aggregates formed with a GICs are of comparable or much smaller size $\left(R_{\mathrm{h}} \sim 6-22 \mathrm{~nm}\right)$. We conclude that if the

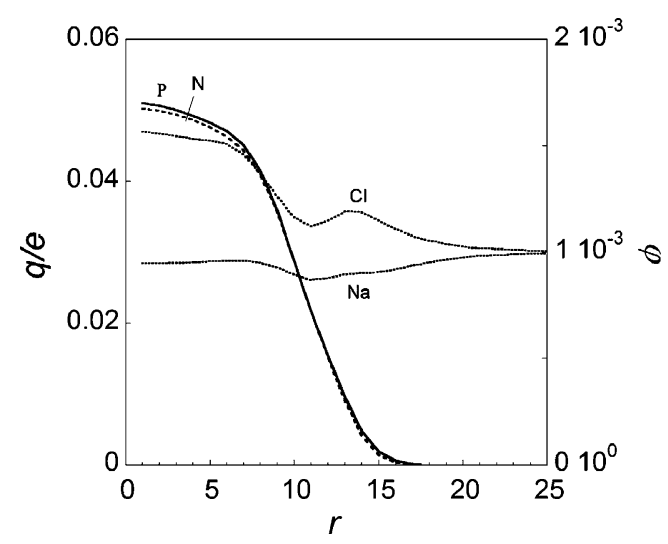

Fig. 17 The dimensionless charge distribution due to the polycation (solid) and polyanion block (dashed), left ordinate, and the radial volume fraction, $\varphi$, profiles for the 1:1 electrolyte ions as indicated. For the parameters, see Table 1 . Volume fraction of salt is $\varphi_{\mathrm{s}}=0.001$ corresponding to the concentration of approximately $0.05 \mathrm{~mol} / \mathrm{l}$ 
aggregates are formed with a GBICs a core-shell structure is formed similar to regular $\mathrm{C} 3 \mathrm{Ms}$, but the hydrophobic endgroups are not (fully) hidden and extend beyond the coronasolvent interface. As a consequence, the assembly of GBICs is driven by two types of interactions: primary assembly is driven by electrostatic attraction between the oppositely charged blocks, and secondary assembly is driven by hydrophobic interactions between the hydrophobic groups at one end of the chains. The grafted copolymer is a relatively stiff molecule in which the charged and neutral monomers are not clearly separated. In GICs, the hydrophobic end-group is expected to be embedded amongst PEO grafts. In this case, the interactions between the hydrophobic chains originating from different aggregates are suppressed for steric reasons, and the assembly is driven mainly by the electrostatic attraction between the oppositely charged groups. The GIC molecules can be thus visualized as polyelectrolyte chains "decorated" with grafts. The applied SCF model cannot account for the secondary assembly between the discussed aggregates originating from the hydrophobic interactions between the hydrophobic end-groups present in copolymer molecules. However, large size disparity between the SCF predictions and the sizes found experimentally for GBICs confirm the possible role of the hydrophobic end-groups in promoting some concentration dependent secondary association. SCF calculations predict that no stable micelles are formed when neutral grafts and charged groups are evenly distributed along the backbone of the copolymer.

Open Access This article is distributed under the terms of the Creative Commons Attribution Noncommercial License which permits any noncommercial use, distribution, and reproduction in any medium, provided the original author(s) and source are credited.

\section{References}

1. Kabanov AV, Bronich TK, Kabanov VA, Yu K, Eisenberg A (1996) Soluble stoichiometric complexes from poly( $N$-ethyl-4vinylpyridinium) cations and poly(ethylene oxide)-block-polymethacrylate anions. Macromolecules 29(21):6797-6802

2. Kataoka K, Togawa H, Harada A, Yasugi K, Matsumoto $T$, Katayose S (1996) Macromolecules 29:8556-8557

3. Brzozowska AM, Zhang Q, de Keizer A, Norde W, Cohen Stuart MA (2010) Protein adsorption on silica and polysulfone surfaces coated with complex coacervate core micelles with poly(vinyl alcohol) as a neutral brush forming block. Colloids Surf A 368(1-3):96-104

4. van der Burgh S, de Keizer A, Cohen Stuart MA (2004) Complex coacervation core micelles. Colloidal stability and aggregation mechanism. Langmuir 20(4):1073-1084

5. Borisov OV, Zhulina EB (2002) Effect of salt on self-assembly in charged block copolymer micelles. Macromolecules 35(11):44724480

6. Brzozowska AM, Hofs B, de Keizer A, Fokkink R, Cohen Stuart MA, Norde W (2009) Reduction of protein adsorption on silica and polystyrene surfaces due to coating with complex coacervate core micelles. Colloids Surf A 347:146-155
7. Postmus BR (2008) Polymer vs. surfactant: competitive adsorption at the solid-liquid interface. Wageningen University, Wageningen, p 144

8. Brzozowska AM, de Keizer A, Detrembleur C, Cohen Stuart MA, Norde W (2010) Grafted ionomer complexes and their effect on protein adsorption on silica and polysulfone surfaces. Colloid Polym Sci 228:1621-1632

9. Brzozowska AM, de Keizer A, Norde W, Detrembleur C, Cohen Stuart MA (2010) Grafted block complex coacervate core micelles and their effect on protein adsorption on silica and polystyrene. Colloid Polym Sci 288:1081-1095

10. Rayleigh L (1910) the incidence of light upon a transparent sphere of dimensions comparable with the wave-length. Proc R Soc Lond A $84: 25-46$

11. Guinier A (1939) Ann Phys 12:161-237

12. Leermakers FAM, Eriksson JC, Lyklema J (2005) Association colloids and their equilibrium modelling, chapter 4. In: Lyklema J (ed) Fundamentals of interface and colloid science, vol. V: soft colloids. Elsevier, Amsterdam. pp. 4.1-4.123

13. Leermakers FAM, Scheutjens JMHM (1990) Statistical thermodynamics of associated colloids. V. Critical micelle concentration, micellar size and shape. J Colloid Interface Sci 160:231-241

14. Leermakers FAM, Wijmans CM, Fleer GJ (1995) On the structure of polymeric micelles - self-consistent-field theory and universal properties for volume fraction profiles. Macromolecules 28(9):3434-3443

15. Sprakel J, Leermakers FAM, Cohen Stuart MA, Besseling NAM (2008) Comprehensive theory for star-like polymer micelles; combining classical nucleation and polymer brush theory. Phys Chem Chem Phys 10(34):5308-5316

16. Lindhoud S, Cohen Stuart MA, Norde W, Leermakers FAM (2009) Self-consistent-field calculations of proteinlike incorporations in polyelectrolyte complex micelles. Phys Rev E Stat Nonlin Soft Matter Phys 80(5):051406

17. Voets IK, Leermakers FAM (2008) Self-consistent field theory for obligatory co-assembly. Phys Rev E Stat Nonlin Soft Matter Phys 78:061801

18. Charlaganov M, Košovan P, Leermakers FAM (2009) New ends to the tale of tails: adsorption of comb polymers and the effect on colloid stability. Soft Matter 5:1448-1459

19. Evers OA, Scheutjens J, Fleer GJ (1990) Statistical thermodynamics of block copolymer adsorption. 1. Formulation of the model and results for the adsorbed layer structure. Macromolecules 23(25):5221-5233

20. Fleer GJ, Cohen Stuart MA, Scheutjens JMHM, Cosgrove T, Vincent B (1993) Polymers at interfaces. Chapman \& Hall, London, p 502

21. Aquil A, Vasseur S, Duguet E, Passirani C, Benoît JP, Roch ARM, Jérôme R, Jérôme C (2008) PEO coated magnetic nanoparticles for biomedical application. Eur Polym J 44:3191-3199

22. Hofs B, Brzozowska A, de Keizer A, Norde W, Cohen Stuart MA (2008) Reduction of protein adsorption to a solid surface by a coating composed of polymeric micelles with a glass-like core. J Colloid Interface Sci 325(2):309-315

23. Hiemenz PC, Lodge TP (2007) Polymer Chemistry, 2nd edn. CRC Press, Boca Raton, p 587

24. Hall DG, Pethica BA (1976) Non-ionic Surfactants, chapter 16. Marcel Dekker, New York

25. Hill TL (1991) Thermodynamics of small systems, part 1. Dover Publications, New York

26. Hill TL (1992) Thermodynamics of small systems, part 2. Dover Publications, New York

27. Leermakers FAM, Lyklema J (1992) On the self-consistent fieldtheory of surfactant micelles. Colloids Surf 67:239-255

28. Voets IK, de Keizer A, Cohen Stuart MA (2009) Complex coacervate core micelles. Adv Colloid Interface Sci 147-48:300-318

29. Postmus BR, Leermakers FAM, Cohen Stuart MA (2008) Selfconsistent field modeling of adsorption from polymer/surfactant mixtures. Langmuir 24(13):6712-6720 